\title{
Electrical modalities beyond pacing for the treatment of heart failure
}

\author{
Richard N. Cornelussen - Vincent Splett • \\ Ruth Nicholson Klepfer · Berthold Stegemann • \\ Lilian Kornet • Frits W. Prinzen
}

Published online: 23 November 2010

(C) The Author(s) 2010. This article is published with open access at Springerlink.com

\begin{abstract}
In this review, we report on electrical modalities, which do not fit the definition of pacemaker, but increase cardiac performance either by direct application to the heart (e.g., post-extrasystolic potentiation or nonexcitatory stimulation) or indirectly through activation of the nervous system (e.g., vagal or sympathetic activation). The physiological background of the possible mechanisms of these electrical modalities and their potential application to treat heart failure are discussed.
\end{abstract}

Keywords Pacing $\cdot$ Nervous system $\cdot($ Electro)

Physiology $\cdot$ Contractility $\cdot$ Heart failure

\section{Introduction}

The use of electrical therapy for cardiac rhythm disturbances is acknowledged for decades, with a primary emphasis on pacing for rate support (e.g., atrioventricular block or sinus node disease), cardiac resynchronization therapy (CRT), and implantable cardioverter-defibrillators (ICD). This review will focus on less well-known electrical therapies for treatment of heart failure (HF) or for prevention of the progression into heart failure. These

R. N. Cornelussen $(\bowtie) \cdot$ B. Stegemann $\cdot$ L. Kornet Research and Technology, Medtronic Bakken Research Center BV, Endepolsdomein 5, $6229 \mathrm{GW}$ Maastricht, The Netherlands e-mail: richard.cornelussen@medtronic.com

V. Splett $\cdot$ R. N. Klepfer

CRDM, Medtronic Moundsview, Minneapolis, MN, USA

R. N. Cornelussen · F. W. Prinzen

Department of Physiology, Maastricht University, Maastricht, The Netherlands therapies are either in their infancy or not yet approved for the treatment of HF. Some discussed therapies might be more beneficial for HF secondary to prior infarction, while other therapies might be more applicable to HF linked to other pathologies such as hypertension or diabetes.

A decrease in contractility is undoubtedly a major contributing factor in the pathophysiology of a significant portion of the patients with heart failure. A reasonable therapeutic goal in these patients would then be to increase cardiac contractility either acutely or long term. The chronic use of inotropic drugs to treat chronic HF has fallen out of favor with the medical community because of the increased mortality seen with these drugs in large clinical trials $[1,2]$. The use of novel stimulation therapies, presented in this review, may offer alternative ways of improving atrial and/or ventricular function. The discussed therapies are divided into electrical stimulation directly on the heart or neural stimulation that affects the heart, such as vagal nerve or spinal cord stimulation. For an overview of the different electrical therapies addressed in this chapter and to know when they are applied within the cardiac cycle, see Fig. 1.

\section{Electrical stimulation on the heart}

Post-extrasystolic potentiation (PESP)

Post-extrasystolic potentiation (PESP) is an intrinsic property of mammalian cardiac muscle, whereby closely spaced depolarizations increase the contractility of the following beat (Fig. 2) [3]. The degree of potentiation produced by an extrasystole depends upon the extrasystolic coupling interval, with increasing potentiation at shorter coupling intervals. In the intact heart, there may be 
Fig. 1 Overview of the timing of the different electrical therapies. Note PESP can also be given after the refractory period of the atrium. This, however, is not depicted in the figure. In some cases, SS was also administered exclusively during the refractory period of the ventricles (see text for details). VS vagal stimulation, SCS spinal cord stimulation, SS sympathetic stimulation

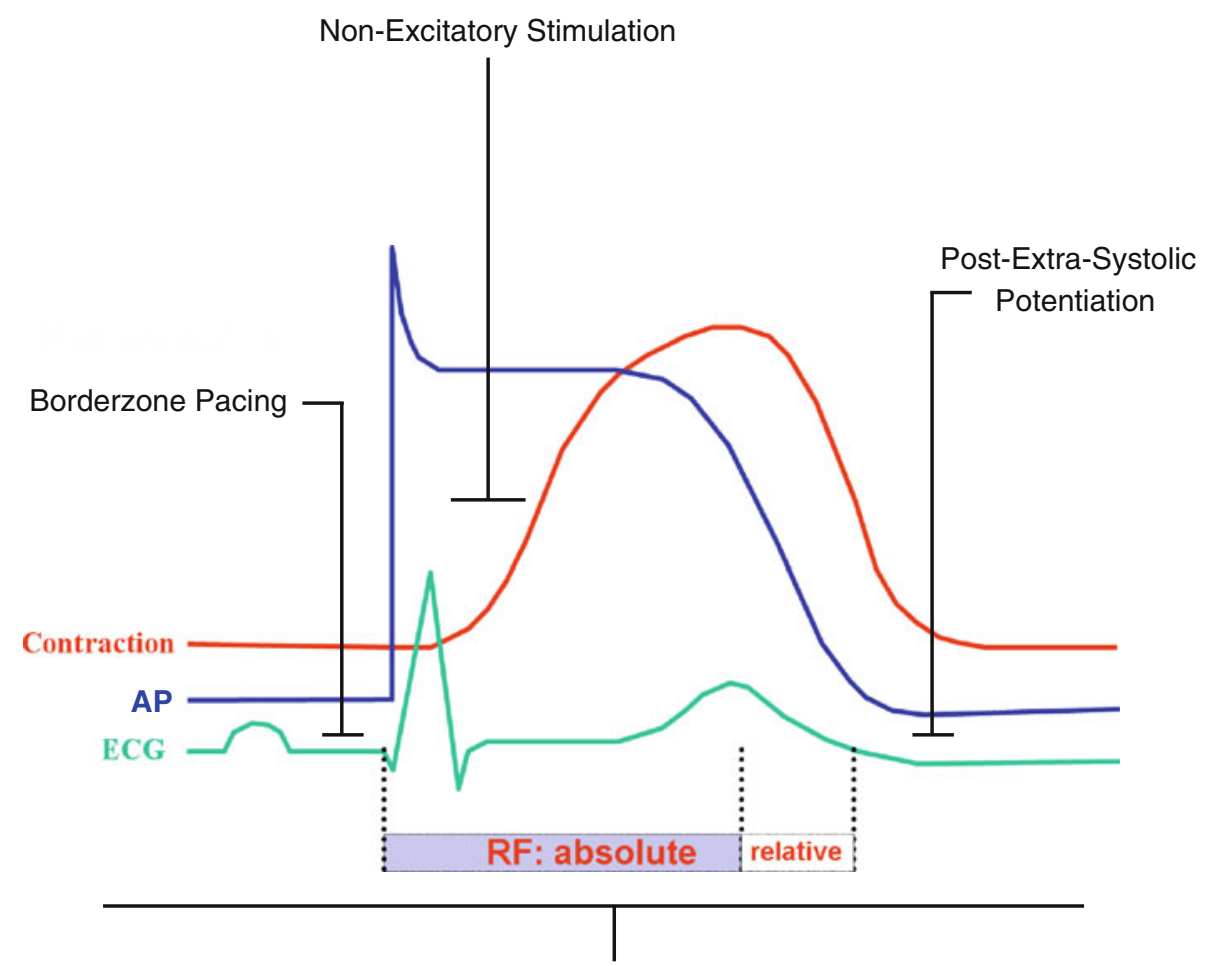

Nerve stimulation (VS, SCS, SS) increased filling after the premature beat, increasing contractile force due to the Frank-Starling effect, but studies in isovolumic hearts and isolated muscle have shown that increased filling has a minor contribution on the magnitude of the effect of PESP [4].

The mechanism responsible for PESP is thought to involve alterations in the uptake and release of $\mathrm{Ca}^{2+}$ from the sarcoplasmic reticulum (SR) [5]. The amount of $\mathrm{Ca}^{2+}$ delivered across the cell membrane into the cell during the depolarization, and the uptake by the SR is relatively normal during the extrasystole. But the extrasystole occurs before the SR has fully recovered and results in reduced SR $\mathrm{Ca}^{2+}$ release, which leads to reduced cytosolic $\mathrm{Ca}^{2+}$ concentration and little developed force (S2 of C in Fig. 2). A relatively normal uptake of $\mathrm{Ca}^{2+}$ occurs during relaxation, but because a less than normal amount of $\mathrm{Ca}^{2+}$ was released from the SR during the extrasystole, the $\mathrm{SR}$ is now loaded with more $\mathrm{Ca}^{2+}$ than normal. The result is that on the following beat, the SR releases more $\mathrm{Ca}^{2+}$ than normal, resulting in a stronger contraction ( $\mathrm{S} 3$ of $\mathrm{C}$ in Fig. 2). The extrasystole generally does not develop enough pressure to eject blood into the aorta.

PESP can be delivered in either a coupled pacing mode, where premature stimuli are delivered after every intrinsic ventricular depolarization, or a paired pacing mode, where premature stimuli are delivered after every ventricular pace. An enhancement to coupled pacing is to deliver it in a dual-chamber mode, where both the atrium and ventricles are prematurely depolarized. Dual-chamber coupled pacing (DCCP) may improve hemodynamics over coupled pacing levels by also enhancing atrial transport function through atrial PESP.

\section{Clinical applications}

There was intense interest in the acute effects of paired/ coupled pacing on cardiac performance in both animals and patients from approximately 1960 to 1970 [6-9]. A canine study in 1966 showed that paired pacing has little effect on baseline cardiac output or mean arterial blood pressure, but when ventricular contractility is depressed by a high dose of pentobarbital, paired pacing increases cardiac output and blood pressure [8]. External pacemakers were developed in the 1960s that performed both coupled and paired ventricular pacing, and the first published record of coupled pacing in a human subject was performed by Eugene Braunwald [6] in the cardiac catheter lab in 1964. An early clinical study in 7 patients with cardiogenic shock in the presence of acute myocardial infarction showed that coupled pacing dramatically improved cardiac function with a clearing of the shock and/or failure in 4 patients [10]. In that series, coupled pacing did not provoke ventricular arrhythmias, whereas paired pacing caused repetitive ventricular responses in $2 / 3$ cases in which it was applied. Despite the early enthusiasm in paired/coupled pacing as a means of improving contractility, the interest in this type of 
(a) S1-S2 interval long

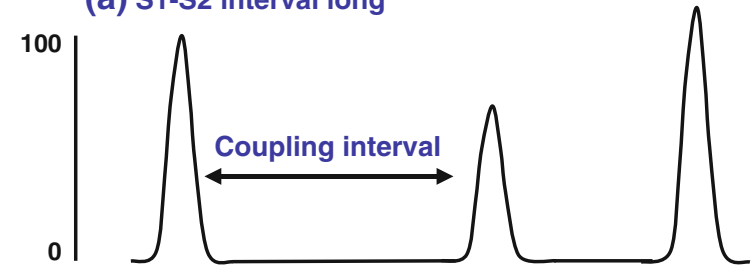

(b) S1-S2 interval medium

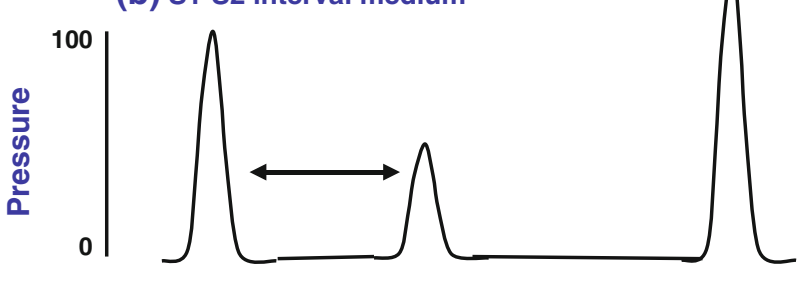

(c) S1-S2 interval short

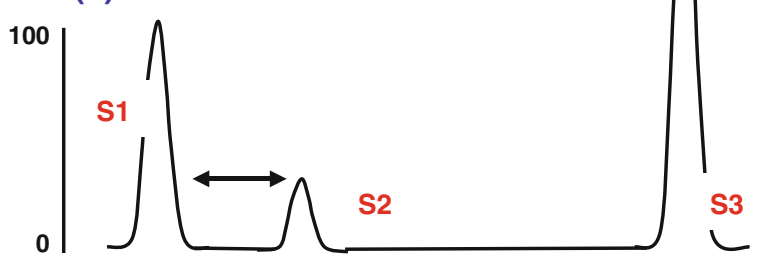

Fig. 2 Example of the concept of post-extrasystolic potentiation. The coupling interval represents the time difference between the first and second depolarization and is ECG-based. The shorter the coupling interval, the bigger the contractility increase of the $S 3$, but the smaller the amplitude of the $S 2$. The drop in mechanical heart rate is similar. The $y$-axis displays normalized pressure while the $x$-axis displays time

pacing waned in the 1970s for unknown reasons. Concerns of pro-arrhythmia and increased metabolic demand were emphasized in several of the early publications $[7,9]$.

We have recently evaluated the effects of coupled pacing in patients with heart failure [11, 12]. In 16 subjects with NYHA class II or III symptoms and an ejection fraction (EF) $<35 \%$, DCCP was acutely applied. The ventricular-coupled paces were delivered $10 \mathrm{~ms}$ after the individually measured effective refractory period $(\mathrm{ERP}=321 \pm 41 \mathrm{~ms})$, and atrial rate support at $45 \mathrm{bpm}$ (excluding the intentional premature beats) was provided during DCCP, if necessary. Compared to normal sinus rhythm (NSR), DCCP increased LV dP/dt max., a surrogate for contractility, by $33 \pm 15 \%$ and arterial pulse pressure by $35 \pm 24 \%$ (see Fig. 3 ). There were no significant changes in mean arterial pressure, mean pulmonary artery pressure, cardiac output, or mixed venous $\mathrm{O}_{2}$ saturation. DCCP as delivered in this study resulted in a decrease in heart rate $(29 \pm 12 \%)$. The increase in LV dP/ dt max was substantially less than the $72 \%$ increase in $\mathrm{LV}$
$\mathrm{dP} / \mathrm{dt}$ max than reported in a previous study by Geschwind et al. that evaluated the hemodynamic effects of $10 \mathrm{~min}$ of $\mathrm{RV}$ coupled pacing in 30 patients with normal LV function $(E F>45 \%)$ [13]. The explanation for the lower-thanexpected coupled pacing induced increase in contractility in our study is not clear. It is possible that the chronic use of drugs such as beta blockers or angiotensin-converting enzyme inhibitors altered the response of HF patients to coupled pacing. A second possibility is that the increase in contractility in HF patients may have been attenuated due to sarcoplasmic reticulum (SR) dysfunction. Since PESP relies on the function of the SR, it may well be that the SR dysfunction in $\mathrm{HF}$ attenuates the ability of coupled pacing to increase contractility. An additional observation in the Geshwind study was that during coupled pacing, arterial and coronary plasma norepinephrine levels increased, suggesting sympathetic nervous system activation. This may have been due to a decrease in mean arterial pressure of $13 \%$ in that study.

Studies have shown that ventricular coupled pacing may provide effective rate control and improve hemodynamics during rapidly conducted atrial fibrillation [14] or ventricular tachycardia [15]. However, the benefit is likely due to a reduction in ventricular rate rather than an improvement in the intrinsic contractility.

\section{Non-excitatory stimulation (NES)}

Electrical stimulation of the myocardium during the refractory period (see Fig. 1) can also result in an increase in contractility. The increase in contractility is a function of the amplitude of the stimulation and the location of the stimulating electrodes [16-18]. The stimulation is not conducting - it does not depolarize the myocardium. Therefore, we will refer to this type of stimulation as nonexcitatory stimulation or NES. Different trains of pulses can be applied. The two modes described in this review are depicted in Fig. 4 and are referred to as monophasic or biphasic trains.

\section{Preclinical results}

Early research in NES was focused on understanding how this stimulation interacted with the heart and the autonomic nervous system. In the 1960s and 1970s, there were various studies conducted, which demonstrated the effects of electrical stimulation on both the parasympathetic and sympathetic innervation on different structures of the heart (sinoatrial node, atrioventricular node [19], ventricles [20]). In 1966, Blinks et al. [16] showed that field stimulation of isolated heart muscle increased the release of autonomic transmitters in a stimulus-strength-dependent manner. These data support the idea that the inotropic 
Fig. 3 Actual human data displaying the PESP response [own observations]. The onset of dual chamber coupled pacing in a representative human experiment. The analog records are arranged in the following order: atrial electrogram (AEGM), ventricular electrogram (VEGM), ECG (lead II), aortic blood pressure (ARTP), and left ventricular pressure (LVP). The large stimulus artifacts in the atrial and ventricular electrograms show the timing of the atrial and ventricular paced events. Coupled pacing is depicted on the right. It clearly displays the fusion of the intrinsic beat and the extrasystole and the relatively large extrasystole amplitude (see text for details)

Fig. 4 Waveforms used for NES. The differences between the waveforms applied for nonexcitatory stimulus.

a monophasic train and b biphasic train
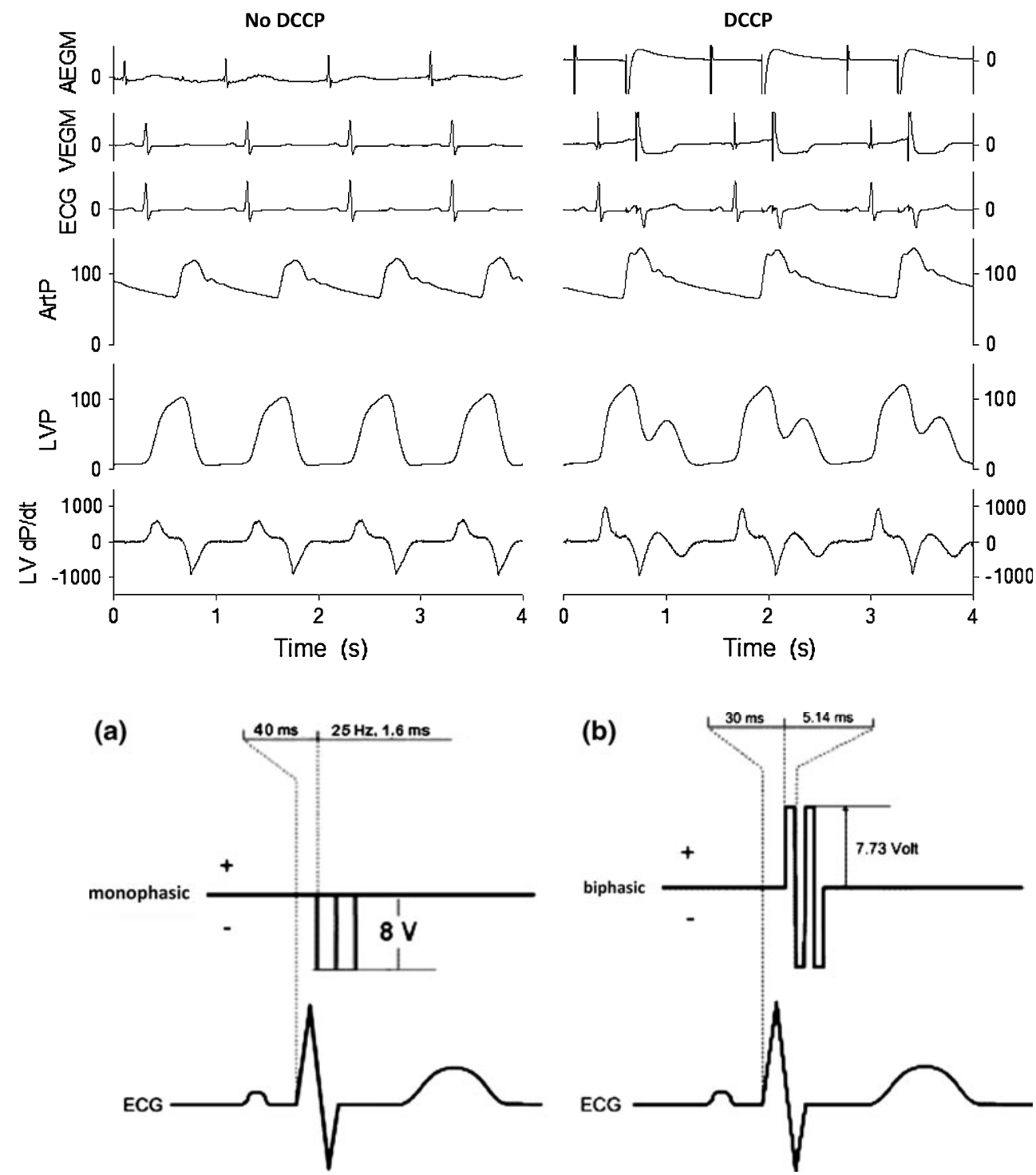

response to NES is mediated by the sympathetic nervous system.

In contrast, in isolated tissue and papillary muscle, the dominant mechanism for increased force generation during NES appears to be field stimulation of the cardiac cells, which alters $\mathrm{Ca}^{2+}$ dynamics. In isolated papillary muscles, both cathodic and anodic NES stimulation have been applied [21]. During cathodic stimulation (negative amplitude), action potential duration and developed force $(-17 \pm 3 \%)$ decreased, while anodic stimulation (positive amplitude) increased both (developed force by $68 \pm 15 \%$ ). These opposite effects of anodic and cathodic NES have been attributed to differential effects on intracellular calcium availability during the contraction, either through altered SR-calcium release or changed cytosolic calcium extrusion [21]. Additional tests of the anodic stimulation in the presence of $\mathrm{Ca}^{2+}$ channel blockers, $\beta$-adrenergic receptor blocker, and ryanodine supported the hypothesis that monophasic stimulation impacts $\mathrm{Ca}^{2+}$ dynamics, in particular by loading the sarcoplasmic reticulum with more $\mathrm{Ca}^{2+}$ to be released on the subsequent beat, similar to PESP (see above). Stimulation of (sympathetic) nerves as a mechanism of changing contractility during anodic or cathodic stimulation was excluded on basis of experiments using propranolol or timing between the observed effect and start of stimulation [21]. However, full beta blockade was not demonstrated, and high energy stimulation of papillary muscles in vitro may, in fact, stimulate the nerves [22].

When studied in intact hearts, the data on the mechanism of NES are mixed. During application of NES in anesthetized, healthy canines contractility increased in the region near the stimulating electrodes and norepinephrine levels increased [17]. The increase in contractility was abolished by complete beta blockade and when the heart was denervated. In our lab, we have reproduced these 
results showing an increase in $\mathrm{LV} \mathrm{dP} / \mathrm{dt} \max$ of $14.1 \pm 8.2 \%$ during NES stimulation with leads placed in the RV apex and anterior-interventricular vein that was significantly decreased to $1.4 \pm 1.0 \%$ by beta blockade with metoprolol ( $1 \mathrm{mg} / \mathrm{kg} \mathrm{IV}$ ) (see Fig. 5). In a separate study in healthy dogs, we further demonstrated that cardiac norepinephrine spillover increased fourfold during NES suggesting sympathetic involvement (see Fig. 6). These results are in contrast to a study done in isolated ferret hearts by Mohri et al. [23]. In this study, NES (biphasic square wave, $\pm 20 \mathrm{~mA}$ ) resulted in an increase in peak-free intracellular calcium $\left(\left[\mathrm{Ca}^{2+}\right]_{\mathrm{i}}\right)$ and increased LV pressure. After the application of $1 \mathrm{mg} / \mathrm{l}$ of propranolol (a sympathetic blocker), the increase in both $\left[\mathrm{Ca}^{2+}\right]_{\mathrm{i}}$ and LV pressure remained, albeit due to NES smaller than if no propanolol was added before beta blockade.

In another dog study, Mohri et al. also demonstrated that application of NES on the LV anterior wall in canines increased contractility in the anterior region of the heart and decreased contractility in the posterior region [18]. These results were reversed when NES was applied in the posterior region. However, the effects of NES on global contractility, as measured by $\mathrm{LV} \mathrm{dP/dt} \mathrm{max,} \mathrm{were} \mathrm{positive}$ during NES $(23.3 \pm 12 \%, 26.0 \pm 9.3 \%$, and $31.3 \pm 12.5 \%$ for anterior, posterior, and both respectively). Acute improvements in global functional measures have also been demonstrated in dogs with LV dysfunction (through serial coronary micro-embolizations) using the biphasic

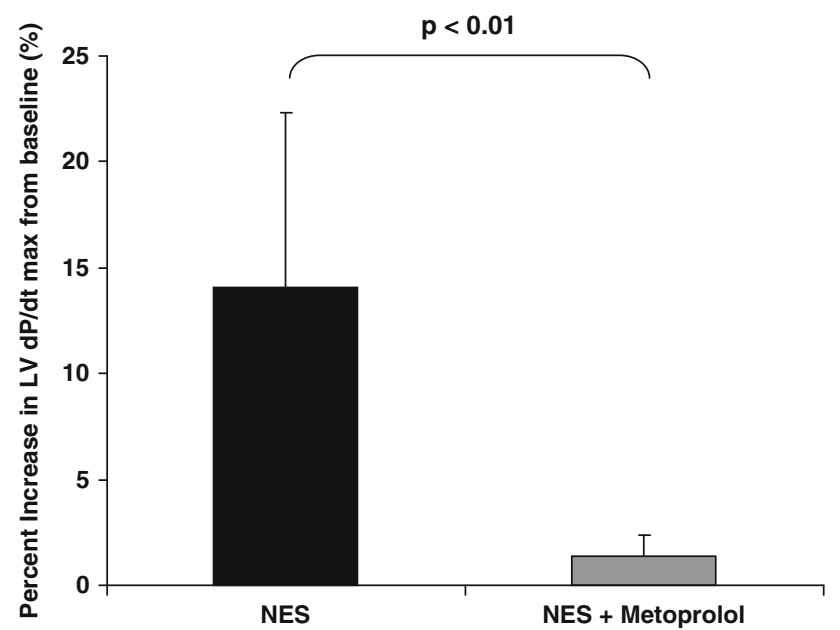

Fig. 5 Effects of metoprolol on the increase of LV dP/dt max during NES. Data from 7 anesthetized animals ( 4 dogs, 3 pigs). NES was delivered through $1 \mathrm{RV}$ lead and $1 \mathrm{LV}$ lead as a biphasic square wave $( \pm 8 \mathrm{~V}) 40 \mathrm{~ms}$ after the sensed ventricular event. Stimulation was applied for $5 \mathrm{~min}$ with $5 \mathrm{~min}$ of normal sinus rhythm before and after stimulation. The median of the last minute of stimulation was used to measure $\mathrm{LV} \mathrm{dP} / \mathrm{dt}$ max. There was a significant reduction in response to NES after the application of metoprolol with no significant change in heart rate or blood pressure. Values plotted are mean \pm standard deviation

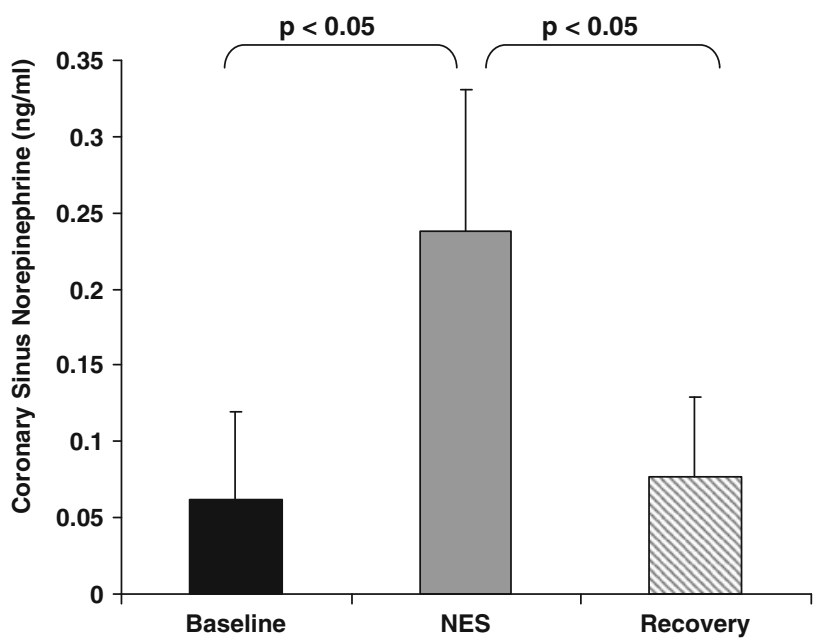

Fig. 6 Coronary sinus catecholamine concentrations during NES. NES was delivered with a monophasic pulse train. Stimulation was applied continuously for $10 \mathrm{~min}$. This was preceded by a 10 -min baseline period and followed by a 10-min recovery period. Coronary sinus and arterial blood samples were withdrawn $(10 \mathrm{ml})$ at the end of each 10-min period and analyzed for epinephrine and nor-epinephrine by radioimmunoassay. Coronary sinus epinephrine did not show a significant change during NES

waveform after $10 \mathrm{~min}$ to $6 \mathrm{~h}$ of stimulation $[24,25]$ and more recently in rabbits with heart failure due to myocardial infarction [26].

We investigated the effect of electrode positioning on the response to NES [27]. NES was delivered with unipolar, epicardial leads on three locations (proximal, mid, and distal) along the left anterior descending (LAD), left circumflex (LCX), and right coronary artery (RCA) acutely in canines. The more proximal the stimulation site was, the larger was the increase in $\mathrm{LV} \mathrm{dP} / \mathrm{dt}$ max, and was largest during proximal LAD NES $(14 \pm 7 \%)$. NES at the RCA sites had no effect on LV dP/dt max, but strongly enhanced $\mathrm{RV} \mathrm{dP} / \mathrm{dt}$ max, indicating the localized effect of NES application. The extent of increase in $\mathrm{LV} \mathrm{dP/dt} \mathrm{max} \mathrm{when}$ during LAD or LCX NES was accompanied by a similar degree of impairment of LV relaxation (e.g., $\mathrm{LV} \mathrm{dP/dt} \mathrm{min}$ decreased by $11 \pm 7 \%$ during proximal LAD NES). During prolonged NES delivery $(1 \mathrm{~h})$, the contractility augmentation significantly decreased from $11 \pm 12 \%$ to $6 \pm 4 \%$. This was accompanied by a corresponding drop in NA-release into the coronary sinus (Fig. 7). These data support the link between the increase in $\mathrm{LV} \mathrm{dP} / \mathrm{dt}$ max and the direct activation of the sympathetic nervous system.

NES has been applied chronically in HF dogs. NES was applied intermittently $(6 \mathrm{~h} /$ day $)$ to the $\mathrm{LV}$ via two coil electrodes in the great cardiac vein for 3 months [24]. The animals receiving NES showed a significant decrease in LVEDP and a significant increase in SV. However, there was also an increase in LVEDV (continued remodeling) and a lack of increase in $\mathrm{LV} \mathrm{dP/dt} \mathrm{max.} \mathrm{The} \mathrm{non-treated}$ 

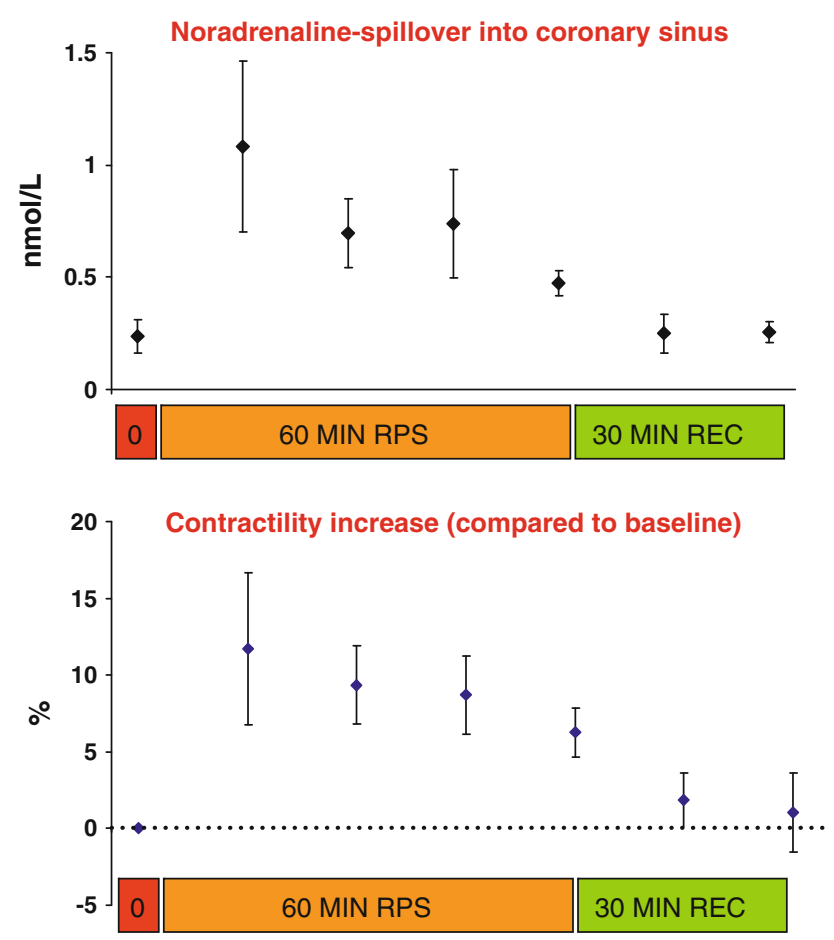

Fig. 7 Extended application of monophasic NES. The effect of $60 \mathrm{~min}$ of monophasic NES ( 3 pulses, $8 \mathrm{~V}, 1.5 \mathrm{~ms} /$ pulse; $40 \mathrm{~ms}$ after the $\mathrm{R}$ wave via one epicardial patch electrode in 5 anesthetized dogs) and 30 min recovery (REC) on normalized changes in LV dP/dt max and the release of nor-adrenaline into the coronary sinus. Data of contractility were normalized to the baseline measurements just before the onset of stimulation. Values are presented as mean \pm SEM. During prolonged RPS delivery, the contractility augmentation significantly decreased from $11 \pm 16 \%$ to $6 \pm 2 \%$. This was accompanied by a similar drop in NA-release into the coronary sinus from $1.08 \pm 0.38$ to $0.47 \pm 0.06 \mathrm{nmol} / \mathrm{l}$ (baseline $0.24 \pm 0.07 \mathrm{nmol} /$ 1). Therapy was delivered unipolar with one lead placed on the basal anterior site (close to the LAD)

dogs worsened in many of these measures, however. Later studies showed changes in gene and protein expression related to $\mathrm{Ca}^{2+}$ dynamics. The treated animals demonstrated significantly different levels of gene and protein expression from the controls, and the changes were in the direction of a non-diseased expression [28, 29]. In another study in dogs with HF, 3 months of NES in dogs with HF shifted protein expression of cytoskeletal proteins and matrix metalloproteinases from pathological toward normal levels [30].

\section{Clinical applications}

The acute effects of NES have been evaluated in 18 patients with heart failure and a narrow QRS complex [31]. Two multipolar catheters were placed in the RV, and a LVpacing lead was placed in a coronary vein. NES was delivered using various electrode configurations including dual- RV site and LV-only NES. An increase in LV dP/dt max greater than 5\% was achieved in 7 (39\%) patients, of whom 4 had LV NES and 3 had dual-RV site NES. No patient discomfort was observed during NES delivered via the RV. Ten patients experienced chest discomfort during NES delivered via the LV and required a reduction in stimulation amplitude.

In addition to this acute data in human HF, biphasic NES therapy has been studied in several other feasibility as well as in chronic studies [31, 32]. Uncontrolled feasibility studies have demonstrated the safety of the therapy [31], and more recently, randomized controlled non-blinded pivotal studies have been conducted in Europe (FIX-HF-4) and in the United States (FIX-HF-5).

Evidence of remodeling and effects on global contractility due to NES have been reported in a non-controlled unblinded feasibility study of 30 class II HF subjects [33]. LV size was measured using three-dimensional echocardiography. Contractile function was assessed using tissue Doppler imaging. NES therapy was applied for 3 months in all subjects. There was a significant decrease in LV ESV and increase in $\mathrm{EF}$ compared to baseline. In addition, peak systolic velocity was increased in all six basal LV segments, even those segments distal to the delivery electrode. Global contractility improved also. There was an improvement in NYHA functional class and 6-minute hall walk as well. Although this study was not controlled or blinded, these data support testing NES in a larger randomized trial.

NES was also studied in a small group of patients ( $N=16)$ who did not respond to CRT [32]. Subjects were followed an average of $147 \pm 80$ days, while several complications such as lead dislocation and appropriate defibrillation were observed, there also was an improvement in NYHA class and ejection fraction. In addition, no electrical interference between the ICD and the NES system was observed.

FIX-HF-4 was a randomized single-blind, cross-over study in which patients received 3 months of active therapy and 3 months received no therapy [34]. Two leads were implanted in the RV, and NES was applied intermittently for a total of $7 \mathrm{~h}$ a day. The primary endpoints were peak $\mathrm{VO}_{2}$ and the MNLWHF quality of life (QOL) questionnaire. Both of these measures improved similarly for the control and therapy groups in the first 3 months of the study. After the cross over, the group that went from $\mathrm{ON}$ to OFF showed a worsening $\mathrm{VO}_{2}$ peak and QOL, while the group that went from OFF to ON showed a very small improvement in both measures. These data suggest a moderate difference between the two groups by the end of the study. In 11 patients, endomyocardial biopsies were obtained at baseline and 3 and 6 months thereafter to test the impact of NES signals on myocardial gene expression [35]. The NES signals were delivered in random order of 
ON for 3 months and OFF for 3 months. The NES signal treatment reverses the cardiac maladaptive fetal gene program and normalizes expression of key sarcoplasmic reticulum $\mathrm{Ca}^{2+}$ cycling and stretch response genes. These changes may contribute to the clinical effects of NES.

In the FIX-HF-5 study, conducted in the United States, patients were randomized to optimal medical therapy (OMT) or OMT plus NES therapy and followed for 12 months [36]. The study was unblinded due to the control group not receiving an implant. Results have not yet been published in a peer-reviewed journal, but were presented orally at the American College of Cardiology conference in 2009 [37]. The study enrolled 428 NYHA class III-IV subjects with EF $\leq 35 \%$ and a narrow QRS [38]. NES was delivered intermittently for $5 \mathrm{~h}$ /day. The primary efficacy endpoint of ventilatory anaerobic threshold (VAT) was not met. However, secondary endpoints of $\mathrm{VO}_{2}$ peak, quality of life, and NYHA class improved significantly. Subgroup analysis suggested that the therapy may be best suited to benefit NYHA III subjects with an EF $\geq 25 \%$, as VAT increased significantly in this subgroup.

Given the data suggesting that the mechanism of effect may be stimulation of local sympathetic nerves, there has been concern about this therapy in systolic HF, in which sympatho-mimetic drugs have been proven to be detrimental [2]. It is possible, however, that NES might avoid the issues seen with inotropic drugs because the stimulation is localized to the myocardium and is delivered intermittently. NES does not appear to generate a systemic sympathetic response. In addition, since some inotropic drugs have been shown to increase myocardial oxygen consumption, this also is a concern for NES. In a small study of both dogs and HF patients, however, up to $30 \mathrm{~min}$ of NES was shown to have no effect on myocardial oxygen consumption [39].

While the results of these studies suggest that there may be benefit to the NES therapy, the strong beneficial effect in the control group and the lack of meeting the primary endpoint in the pivotal suggest that additional studies are needed to find the optimal patient population for this therapy.

\section{Infarct borderzone pacing}

Left ventricular remodeling is a well-known complication after an acute myocardial infarction, which can progress into heart failure. Peri-infarct (borderzone) pacing has recently been proposed as a therapy for the prevention of adverse remodeling [40]. This peri-infarct borderzone pacing is intended to deliberately induce dyssynchrony, which is in contrast to CRT, which pre-excites the endogenously late activating (lateral) segments of the left ventricle, which results in a more synchronous contraction of the ventricle. Remodeling initiated by increases in (systolic) wall stress in the infarcted area can be counteracted by electrical pre-excitation and subsequent unloading of that region. The hypothesis is based upon previous work in normal animals that showed that pacing-induced preexcitation decreased strain and myocardial work at the pacing site and increasing strain distal to it [41]. This concept of regional unloading is in line with benefits of global left ventricular unloading, such as by cardiac assist devices or vasodilators [42-44].

Shuros et al. investigated the optimal pacing site and optimal AV-delay to deliver borderzone pacing in acute experiments in pigs by local strain and stroke work parameters. An AV interval of $50 \%$ of the intrinsic PR interval was shown to acutely reduce the strain in the ischemic borderzone without significantly impeding cardiac output [40]. These pigs were then chronically stimulated and followed for 2 months. Chronic application of pre-excitation pacing attenuated adverse remodeling over time as evidenced by the morphometric data on LV and LA size. Soon after, Saba et al. [45], using small animals with a posterolateral infarct, showed prevention of adverse electrical and mechanical cardiac remodeling with $\mathrm{BiV}$ pacing compared with right ventricular pacing or no pacing after MI.

More insight into the cellular mechanism of borderzone pacing may be provided by a recent study in which regional early LV activation, as in pre-excitation, was also able to change matrix metalloproteinase (MMP)-activity [46, 47]. MMPs are a family of matrix proteases implicated in adverse LV remodeling through matrix remodeling [48]. Other studies have shown that localized high frequency (non-capturing) stimulation within the infarct prevented the thinning of this area and attenuated left ventricular dilatation [49], being associated with beneficial decreased changes in matrix metalloproteinase (MMP) activity as well. This would corroborate the involvement of MMPs in electrical borderzone stimulation. Also, electrical stimulation of its own accord can induce beneficial gene and protein expression [50].

\section{Clinical applications}

Infarct borderzone pacing is just beginning to be explored in humans. In a small pilot clinical study, patients with reduced EF secondary to myocardial infarction were randomized to either biventricular borderzone (BiV) pacing with ICD or ICD alone within 30-45 after MI [51]. After 12 months, LV size was unchanged in the $\mathrm{BiV}$ pacing group while it increased in the ICD-only group. These positive results prompted a larger study, called the MENDMI study [52]. This study evaluated biventricular borderzone pacing in a narrowly defined patient population 
with the borderzone pacing therapy delivered within 14 days after myocardial infarction. The recently published results show that the primary endpoint (change in LV enddiastolic volume after 12 months therapy) was not met [53] (study identifier: NCT00605631). Possible explanations for the discrepancy between this trial and the earlier results might be the relatively low $\mathrm{LV}$ remodeling or an inefficacious therapy delivery. The therapy is a pathophysiologically based approach and needs further investigation.

\section{Electrical stimulation outside the heart}

In the previous paragraphs, we have discussed the application of electrical stimulation on the heart, which has the advantage of avoiding possible adverse side-effects as regularly encountered with pharmacological therapies. The therapies discussed below are applied outside the heart and usually are applied continuously throughout the cardiac cycle (see Fig. 1). Their benefit may be that the stimulation is affecting a much more integrated system. Studies of these therapies clearly show cardiac benefit, but in most cases, the exact mechanism is unknown. This is likely due to multi-organ and central nervous system pathways.

\section{Vagal nerve stimulation}

There has been extensive research demonstrating that acute vagus nerve stimulation results in a decrease in various measures of ventricular function including contractility. Lewis et al. showed that in the human and pig heart, stimulation of the left vagus nerve can profoundly decrease contractility of the left ventricular myocardium, independent of its braducardic effect [54]. This decrease in ventricular contractility during vagal stimulation (VNS) appears to be mediated by the parasympathetic ganglia located in the cranial medial ventricular fat pad $[55,56]$. However, at low sympathetic tone, the negative inotropic effect of vagal stimulation is attributable primarily to its negative chronotropic effect [57]. This suggests that the effect of VNS on contractility is mediated via an interaction with the sympathetic system.

It may seem counterintuitive that a reduction in contractility by VNS may be beneficial to patients with heart failure. However, several pre-clinical studies have shown benefit in chronic vagus nerve stimulation in models of systolic heart failure [58]. Recently, Zhang et al. evaluated VNS in a canine high-rate pacing-induced model of heart failure. VNS at an intensity that reduced sinus rate by approximately $20 \mathrm{bpm}$ was delivered in the VNS group. After 4 and 8 weeks, both left ventricular end-diastolic and end-systolic volumes were lower, and left ventricular EF was higher in the VNS group than in the control group [59].
Li et al. showed that VNS markedly improved the longterm survival of chronic heart failure rats through the prevention of pumping failure, remodeling, and increasing contractility [60]. Very recently, the same group showed that VNS applied immediately after MI attenuated LV remodeling, which may be related to the decreased acute inflammatory response or to the reduction in infarct size induced by VNS [61], since the remodeling process increases with a larger infarct.

\section{Clinical applications}

A phase I clinical study has been conducted in Europe demonstrating the safety and feasibility of chronic vagus nerve stimulation in systolic heart failure patients. The system, CardioFit (BioControl Medical), is an implantable device and lead delivering pulses synchronous with the cardiac cycle to the right cervical vagus nerve. A cohort of 30 patients has been reported on. After 3 months of VNS, the subjects had significant improvements in NYHA class, Minnesota quality of life, QOL, (from $52 \pm 14$ to $31 \pm 18$ ), left ventricular end-systolic volume (from $208 \pm 71$ to $190 \pm 83 \mathrm{ml}$ ), and a favorable trend toward reduction in end-diastolic volume [62]. After 6 months of VNS, EF, 6-min hall walk, and QOL had significantly improved. These encouraging results need to be confirmed in a randomized controlled trial.

\section{Sympathetic stimulation}

Sympathetic-parasympathetic interaction plays a major role in the evolution and outcome of HF. Numerous sympatho-mimetic drug studies have shown acute beneficial but chronic adverse effects. The major concerns with using these drugs are the increased mortality associated with their use, in addition to an increase in cardiac oxygen consumption and side-effects [1]. Sympatho-mimetic electrical therapies may be able to circumvent the adverse profile of sympatho-mimetic drugs by being more selective to sympathetic nerves on the heart and by their intermittent nature.

Selective stimulation of the cardiac sympathetic nerves by transvascular stimulation of the heart has been shown $[63,64]$. Cardiac sympathetic nerves course within neural sleeves along the subclavian artery. Because of this proximity, transvascular sympathetic nerve stimulation was attempted with electrode catheters inside the subclavian artery. Right as well as left subclavian sympathetic nerve stimulation $(20 \mathrm{~Hz})$ during high-rate ventricular pacing evoked a more than $100 \%$ increase of left ventricular systolic pressure. In a separate study [64], sympathetic neural structures inside the heart were identified that selectively control left ventricular inotropy and could be 
accessed by transvenous catheter stimulation. High frequency $(200 \mathrm{~Hz})$ stimulation was applied in the ventricular absolute refractory period $(50 \mathrm{~ms}$ long, $20 \mathrm{~ms}$ after the $\mathrm{R}$ wave; resembling NES; see above) during pacing at $120 \mathrm{bpm}$. Stimulation generated a significant increase in LV dP/dt max $(\sim 50 \%)$, LV peak pressure $(\sim 40 \%)$, cardiac output $(\sim 25 \%)$, and the slope of the end-systolic pressure-volume relationship ( $\sim 35 \%)$, a load independent measure of contractility. The effect was abolished by acute beta blockade, indicating that the effect was likely due to sympathetic nerve simulation. In addition, the transcardiac norepinephrine gradient increased from 0.15 to $3.7 \mathrm{nmol} / 1$ during stimulation.

For comparison, if the stellate ganglion was stimulated, norepinephrine content rose 200 times in the coronary artery when compared to baseline [65], which indicates the relatively gentle and local nature of the above-mentioned transvenous approach to sympathetic stimulation. However, due to the nature of the nerve stimulus (high frequency and throughout the cardiac cycle), adverse events like ventricular extra systoles and atrial or ventricular arrhythmias are anticipated. Further studies are required to show the safety and efficacy of this novel approach.

\section{Spinal cord stimulation}

Thoracic spinal cord stimulation (SCS) has been used to treat angina pectoris and to affect cardiac autonomic balance. Although the precise mechanism is unknown, current evidence suggests that spinal cord stimulation at the T1-T5 level decreases sympathetic tone to the heart [66, 67]. Rebalancing the autonomic nervous system may reduce the systemic release of cathecholamines, reduce sympathetic activation of the renin-angiotension system in the kidneys and have an anti-arrhythmic effect [68]. In addition, spinal cord stimulation might activate large afferent fibers; GABAergic connections in the superficial dorsal horn may suppress the processing of information in the spinothalamic tract neurons [69] or via VR-1 containing sensory fibers [70]. There are also effects of SCS seen in the vasculature that could result in benefits in HF. For a full review on the beneficial effects of SCS on the vasculature, see Wu et al. [71].

Lopshire et al. [72] investigated the effects of long-term SCS on ventricular function in a canine post-infarction high-rate pacing model of heart failure. They found that a greater recovery in ejection fraction was noted in the SCS $(52 \pm 5 \%)$ group compared with a group treated by beta blockers +ACE inhibitors $(38 \pm 2 \%)$ and a control $(31 \pm 4 \%)$ group. Furthermore, there was a reduction in serum norepinephrine levels in the SCS-treated dogs, supporting the hypothesis that SCS reduces sympathetic tone.
A world-wide study, Defeat-HF, has recently started enrolling to evaluate the effects of SCS in a systolic heart failure population. It is a prospective, multicenter, randomized, parallel controlled study expected to enroll up to 70 patients, registered under NCT01112579 at www. clinicaltrails.gov.

\section{Considerations and perspectives}

This review has described several electrical therapies that are not used broadly in clinical practice as of today. Adoption of these therapies may be years away given their efficacy, current means of implementation, and the need for additional clinical evidence. However, new electrophysiological knowledge and continuous device development might facilitate the adoption of these therapies in the area of cardiac disease.

Each of the discussed "therapies" may have its pros and cons. With respect to the contractility enhancement therapy involving PESP, the data suggest that chronic application may not be as arrhythmogenic as feared but also that it does not result in a large enough positive hemodynamic response in patients with chronic heart failure. PESP may have benefit in other conditions where sarcoplasmic reticulum function is closer to normal, however. NES may at least partly depend upon the local release of nor-epinephrine. Regardless of the mechanism, additional data showing clear benefit in the heart failure population are necessary for wide-spread adoption. Infarct borderzone stimulation is a patho-physiologically approach based on reducing stress and load. Besides that its benefits have to be better supported by clinical data, the question remains as to whether long-term stretching of the late-activated region might lead to decompensation.

Sympathetic stimulation specifically for the heart may result in controlled modulation of the sympathetic tone of the left ventricle, but energy costs should be evaluated to ensure they are not too high. Mechanistically, vagal stimulation seems to have the greatest potential, because of the strong association between impaired vagal reflexes and increased cardiac mortality [60]. However, clinical data are limited.

Open Access This article is distributed under the terms of the Creative Commons Attribution Noncommercial License which permits any noncommercial use, distribution, and reproduction in any medium, provided the original author(s) and source are credited.

\section{References}

1. Packer M et al (1991) Effect of oral milrinone on mortality in severe chronic heart failure. The PROMISE study research group. N Engl J Med 325(21):1468-1475 
2. O'Connor CM et al (1999) Continuous intravenous dobutamine is associated with an increased risk of death in patients with advanced heart failure: insights from the Flolan International Randomized Survival Trial (FIRST). Am Heart J 138(1):78-86

3. Cooper MW (1993) Postextrasystolic potentiation. Do we really know what it means and how to use it? Circulation 88(6): 2962-2971

4. Kuijer PJ, van der Werf T, Meijler FL (1990) Post-extrasystolic potentiation without a compensatory pause in normal and diseased hearts. Br Heart J 63(5):284-286

5. Gwathmey JK, Hajjar RJ (1990) Relation between steady-state force and intracellular $[\mathrm{Ca} 2+]$ in intact human myocardium. Index of myofibrillar responsiveness to $\mathrm{Ca} 2+$. Circulation 82(4):1266-1278

6. Braunwald E et al (1964) Clinical observations on paired electrical stimulation of the heart. Effects on ventricular performance and heart rate. Am J Med 37:700-711

7. Frommer PL (1965) Studies on coupled pacing technique and some comments on paired electrical stimulation. Bull N Y Acad Med 41:670-680

8. Frommer PL, Robinson BF, Braunwald E (1966) Paired electrical stimulation. A comparison of the effects on performance of the failing and nonfailing heart. Am J Cardiol 18(5):738-744

9. Rothfeld EL et al (1969) Paired pacing after coronary artery ligation. Am J Cardiol 23(2):224-228

10. Gourgon R et al (1968) Ventricular coupled pacing in myocardial infarction with severe cardiocirculatory insufficiency. In: Cranefield P, Hoffman B (eds) Paired Pulse Stimulation of the Heart. The Rockefeller University Press, New York, pp 36-51

11. Lieberman RA et al (2008) The acute hemodynamic response to dual chamber coupled pacing in heart failure patients. J Card Fail 14(6):s27

12. Lieberman RA et al (2008) The acute hemodynamic response to dual chamber coupled pacing in heart failure patients. J Cardiac Failure 14(6):s58

13. Geschwind HJ et al (1984) Sympathetic nervous system activation in postextrasystolic potentiation: role of catecholamine release in enhancement of ventricular function. J Am Coll Cardiol 4(2):216-225

14. Lau CP et al (1990) A new pacing method for rapid regularization and rate control in atrial fibrillation. Am $\mathrm{J}$ Cardiol 65(18):1198-1203

15. Mischke K et al (2006) Paired ventricular stimulation: an approach for hemodynamic stabilization during ventricular tachycardia. J Am Coll Cardiol 47(11):2337-2339

16. Blinks Jr (1966) Field stimulation as a means of effecting the graded release of autonomic transmitters in isolated heart muscle. J Pharmacol Exp Ther 151(2):221-235

17. Euler DE (1980) Release of autonomic neuromediators by local ventricular electrical stimulation. Am J Physiol Heart Circ Physiol 238(6):H794-H800

18. Mohri S et al (2002) Cardiac contractility modulation by electric currents applied during the refractory period. Am J Physiol Heart Circ Physiol 282(5):H1642-H1647

19. Berbari EJ et al (1973) Noninvasive technique for detection of electrical activity during the P-R segment. Circulation 48(5):1005-1013

20. Brady AJ, Abbott BC, Mommaerts WF (1960) Inotropic effects of trains of impulses applied during the contraction of cardiac muscle. J Gen Physiol 44(2):415-432

21. Brunckhorst CB et al (2006) Cardiac contractility modulation by non-excitatory currents: studies in isolated cardiac muscle. Eur J Heart Fail 8(1):7-15

22. Schauerte P et al (2001) Focal atrial fibrillation: experimental evidence for a pathophysiologic role of the autonomic nervous system. J Cardiovasc Electrophysiol 12(5):592-599
23. Mohri S et al (2003) Electric currents applied during refractory period enhance contractility and systolic calcium in the ferret heart. Am J Physiol Heart Circ Physiol 284(4):H1119-H1123

24. Morita $\mathrm{H}$ et al (2004) Long-term effects of non-excitatory cardiac contractility modulation electric signals on the progression of heart failure in dogs. Eur J Heart Fail 6(2):145-150

25. Chaudhry PA et al (2001) Acute ventricular reduction with the acorn cardiac support device: effect on progressive left ventricular dysfunction and dilation in dogs with chronic heart failure. J Card Surg 16(2):118-126

26. Zhang H, Cui C, Hu D (2010) Effects of electric stimulations applied during absolute refractory period on cardiac function of rabbits with heart failure. J Huazhong Univ Sci Technolog Med Sci 30(2):155-158

27. Cornelussen RN et al (2008) Contractility augmentation induced by refractory period stimulation depends upon pacing-site and diminishes over time. Circulation 118:S726

28. Imai $\mathrm{M}$ et al (2007) Therapy with cardiac contractility modulation electrical signals improves left ventricular function and remodeling in dogs with chronic heart failure. J Am Coll Cardiol 49(21):2120-2128

29. Gupta RC et al (2009) Cardiac contractility modulation electrical signals normalize activity, expression, and phosphorylation of the $\mathrm{Na}+-\mathrm{Ca} 2+$ exchanger in heart failure. J Card Fail 15(1):48-56

30. Rastogi $S$ et al (2008) Effects of chronic therapy with cardiac contractility modulation electrical signals on cytoskeletal proteins and matrix metalloproteinases in dogs with heart failure. Cardiology 110(4):230-237

31. Pappone $\mathrm{C}$ et al (2004) First human chronic experience with cardiac contractility modulation by nonexcitatory electrical currents for treating systolic heart failure: mid-term safety and efficacy results from a multicenter study. J Cardiovasc Electrophysiol 15(4):418-427

32. Nägele H, Behrens S, Eisermann C (2008) Cardiac contractility modulation in non-responders to cardiac resynchronization therapy. Europace 10(12):1375-1380

33. Yu C-M et al (2009) Impact of cardiac contractility modulation on left ventricular global and regional function and remodeling. JACC Cardiovasc Imaging 2(12):1341-1349

34. Borggrefe MM et al (2008) Randomized, double blind study of non-excitatory, cardiac contractility modulation electrical impulses for symptomatic heart failure. Eur Heart J 29(8):1019_ 1028

35. Butter C et al (2008) Cardiac contractility modulation electrical signals improve myocardial gene expression in patients with heart failure. J Am Coll Cardiol 51(18):1784-1789

36. Abraham WT et al (2008) A randomized controlled trial to evaluate the safety and efficacy of cardiac contractility modulation in patients with systolic heart failure: rationale, design, and baseline patient characteristics. Am Heart J 156(4):641-648

37. Cleland JGF et al (2009) Clinical trials update from the American College of Cardiology 2009: ADMIRE-HF, PRIMA, STICH, REVERSE, IRIS, partial ventricular support, FIX-HF-5, vagal stimulation, REVIVAL-3, pre-RELAX-AHF, ACTIVE-A, HFACTION, JUPITER, AURORA, and OMEGA. Eur J Heart Fail 11(6):622-630

38. Abraham WT (2009) Multicenter randomized controlled trial of cardiac contractility modulation in patient with advanced heart failure. In: American College of Cardiology Orlando, Florida

39. Butter $\mathrm{C}$ et al (2007) Enhanced inotropic state of the failing left ventricle by cardiac contractility modulation electrical signals is not associated with increased myocardial oxygen consumption. J Card Fail 13(2):137-142

40. Shuros AC et al (2007) Ventricular preexcitation modulates strain and attenuates cardiac remodeling in a swine model of myocardial infarction. Circulation 116(10):1162-1169 
41. Prinzen FW et al (1999) Mapping of regional myocardial strain and work during ventricular pacing: experimental study using magnetic resonance imaging tagging. J Am Coll Cardiol 33(6):1735-1742

42. Heerdt PM et al (2000) Chronic unloading by left ventricular assist device reverses contractile dysfunction and alters gene expression in end-stage heart failure. Circulation 102(22):27132719

43. Jugdutt BI, Butler C (2007) Ventricular unloading tissue angiotensin II, matrix modulation, and function during left ventricular assist device support. J Am Coll Cardiol 49(11):1175-1177

44. Califf RM, O'Connor CM (2000) Beta-blocker therapy for heart failure: the evidence is in now the work begins. JAMA 283(10): $1335-1337$

45. Saba S et al (2008) Prevention of adverse electrical and mechanical remodeling with biventricular pacing in a rabbit model of myocardial infarction. Heart Rhythm 5(1):124-130

46. Mukherjee $\mathrm{R}$, et al. Short-term disruption in regional left ventricular electrical conduction patterns increases interstitial matrix metalloproteinase activity. Am J Physiol Heart Circ Physiol 299(1): H217-24

47. Garcia RA et al (2005) Abnormal cardiac wall motion and early matrix metalloproteinase activity. Am J Physiol Heart Circ Physiol 288(3):H1080-H1087

48. Spinale FG (2007) Myocardial matrix remodeling and the matrix metalloproteinases: influence on cardiac form and function. Physiol Rev 87(4):1285-1342

49. Mukherjee R et al (2010) Long-term localized high-frequency electric stimulation within the myocardial infarct: effects on matrix metalloproteinases and regional remodeling. Circulation 122(1):20-32

50. Brighton CT, Wang W, Clark CC (2008) The effect of electrical fields on gene and protein expression in human osteoarthritic cartilage explants. J Bone Joint Surg Am 90(4):833-848

51. Chung ES et al (2007) Feasibility of biventricular pacing in patients with recent myocardial infarction: impact on ventricular remodeling. Congest Heart Fail 13(1):9-15

52. Chung ES et al (2009) Peri-infarct pacing with CRT in the early postinfarct phase to attenuate long-term remodeling. J Cardiovasc Transl Res 2(1):126-129

53. Chung ES et al (2010) Effect of peri-infarct pacing early after myocardial infarction: results of the prevention of myocardial enlargement and dilatation post myocardial infarction study. Circ Heart Fail 3(6):650-658

54. Lewis ME et al (2001) Vagus nerve stimulation decreases left ventricular contractility in vivo in the human and pig heart. J Physiol 534(Pt. 2):547-552

55. Gatti PJ et al (1997) Vagal control of left ventricular contractility is selectively mediated by a cranioventricular intracardiac ganglion in the cat. J Auton Nerv Syst 66(3):138-144

56. Blinder KJ et al (1998) Control of negative inotropic vagal preganglionic neurons in the dog: synaptic interactions with substance $\mathrm{P}$ afferent terminals in the nucleus ambiguus? Brain Res 810(1-2):251-256
57. Matsuura W et al (1997) Vagal stimulation decreases left ventricular contractility mainly through negative chronotropic effect. Am J Physiol 273(2 Pt 2): H534-9

58. Sabbah $\mathrm{H}$ et al (2005) Long-term therapy with neuroselectiveelectric vagus nerve stimulation improves $\mathrm{LV}$ function and attenuates global LV remodeling in dogs with chronic heart failure. Eur J Heart Fail 166: 166 (Abstract)

59. Zhang $\mathrm{Y}$ et al (2009) Chronic vagus nerve stimulation improves autonomic control and attenuates systemic inflammation and heart failure progression in a canine high-rate pacing model. Circ Heart Fail 2(6):692-699

60. Li M et al (2004) Vagal nerve stimulation markedly improves long-term survival after chronic heart failure in rats. Circulation 109(1):120-124

61. Uemura K et al (2010) Early short-term vagal nerve stimulation attenuates cardiac remodeling after reperfused myocardial infarction. J Card Fail 16(8):689-699

62. De Ferrari GM, Sanzo A, Schwartz PJ (2009) Chronic vagal stimulation in patients with congestive heart failure. Conf Proc IEEE Eng Med Biol Soc 2009:2037-2039

63. Zarse $M$ et al (2005) Selective increase of cardiac neuronal sympathetic tone: a catheter-based access to modulate left ventricular contractility. J Am Coll Cardiol 46(7):1354-1359

64. Meyer C et al (2010) Augmentation of left ventricular contractility by cardiac sympathetic neural stimulation. Circulation 121(11):1286-1294

65. Watson-Wright WM et al (1992) Prolonged supramaximal stimulation of canine efferent sympathetic neurons induces desensitization of inotropic responses without a change in myocardial beta-adrenergic receptors. Can J Cardiol 8(2):177-186

66. Sanderson JE et al (1994) Spinal electrical stimulation for intractable angina-long-term clinical outcome and safety. Eur Heart J 15(6):810-814

67. Eliasson T, Augustinsson LE, Mannheimer C (1996) Spinal cord stimulation in severe angina pectoris-presentation of current studies indications and clinical experience. Pain 65(2-3):169-179

68. Bacal $F$ et al (2005) Normalization of right ventricular performance and remodeling evaluated by magnetic resonance imaging at late follow-up of heart transplantation: relationship between function, exercise capacity and pulmonary vascular resistance. J Heart Lung Transplant 24(12):2031-2036

69. Meyerson BA, Linderoth B (2006) Mode of action of spinal cord stimulation in neuropathic pain. J Pain Symptom Manage 31 (4 Suppl):S6-S12

70. Wu M et al (2006) Sensory fibers containing vanilloid receptor-1 (VR-1) mediate spinal cord stimulation-induced vasodilation. Brain Res 1107(1):177-184

71. Wu M, Linderoth B, Foreman RD (2008) Putative mechanisms behind effects of spinal cord stimulation on vascular diseases: a review of experimental studies. Auton Neurosci 138(1-2):9-23

72. Lopshire JC et al (2009) Spinal cord stimulation improves ventricular function and reduces ventricular arrhythmias in a canine postinfarction heart failure model. Circulation 120(4):286-294 